\title{
ON THE NONCOMMUTATIVE GEOMETRY OF THE ENDOMORPHISM ALGEBRA OF A VECTOR BUNDLE
}

\author{
Thierry MASSON \\ Laboratoire de Physique Théorique et Hautes Energies ${ }^{1}$ \\ Université Paris XI, Bâtiment 211 \\ 91405 Orsay Cedex, France \\ e-mail: masson@qcd.th.u-psud.fr
}

\begin{abstract}
In this letter we investigate some aspects of the noncommutative differential geometry based on derivations of the algebra of endomorphisms of an oriented complex hermitian vector bundle. We relate it, in a natural way, to the geometry of the underlying principal bundle and compute the cohomology of its complex of noncommutative differential forms.
\end{abstract}

L.P.T.H.E.-ORSAY 98/14

${ }^{1}$ Laboratoire associé au Centre National de la Recherche Scientifique - URA D0063 


\section{Introduction and notations}

In [5], it was shown that the noncommutative geometry of the algebra of endomorphisms of an oriented complex hermitian vector bundle is very much like the ordinary geometry of the associated $S U(n)$-principal bundle. In particular, from the point of view of connections, this noncommutative algebra gives us interesting relations with the canonical Atiyah Lie algebroid associated to this oriented vector bundle.

In this letter, we would like to proceed in the study of the noncommutative differential calculus of this endomorphisms algebra. In particular, we would like to make closer relations with the ordinary geometry of the principal bundle. Using ordinary technics in algebraic geometry, we will compute the cohomology of this noncommutative differential calculus.

The notations we will use in this letter are those of [5], and we refer the reader to this paper for more details on the construction and properties of the objects we will introduce here.

Let $M$ be a regular finite dimensional smooth manifold. We denote by $E$ an oriented complex hermitian vector bundle of rank $n$ over $M$, by $P$ its $S U(n)$ principal frame bundle, and we introduce $\mathfrak{A}$ the algebra of endomorphisms of $E$, which is the set of sections of $\operatorname{End}(E)$. We denote by $\left(\Omega_{\operatorname{Der}}(\mathfrak{A}), \hat{d}\right)$ the noncommutative differential calculus based on derivations $[2,6,5]$.

\section{Some relations between $\Omega_{\operatorname{Der}}(\mathfrak{A})$ and $\Omega(P)$}

In this section we would like to give some structure properties of $\Omega_{\text {Der }}(\mathfrak{A})$ which relates it to the ordinary differential calculus $\Omega(P)$ of $P$.

Let us denote by $\mathcal{F}(P)$ the (commutative) algebra of smooth functions on $P$ and by $A \mapsto A^{v}$ the map which sends any $A \in \mathfrak{s u}(n)$ into the associated vertical vector field on $P$.

Let us introduce the algebra $\mathfrak{B}=\mathcal{F}(P) \otimes M_{n}(\mathbb{C})$ of matrix valued functions on $P$. Denote by $\left(\Omega_{\text {Der }}(\mathfrak{B}), \hat{d}\right)=\left(\Omega(P) \otimes \Omega_{\text {Der }}\left(M_{n}(\mathbb{C})\right), d+d^{\prime}\right)$ its differential calculus based on derivations [4]. It is easy to see that $\mathcal{G}=\left\{A^{v}+a d_{A} / A \in \mathfrak{s u}(n)\right\}$ is 
a Lie subalgebra of $\operatorname{Der}(\mathfrak{B})$ isomorphic to $\mathfrak{s u}(n)$. This Lie subalgebra defines a Cartan operation of $\mathfrak{s u}(n)$ on $\Omega_{\text {Der }}(\mathfrak{B})$, whose basic subalgebra we denote by $\Omega_{\text {Der,Bas }}(\mathfrak{B})$. Then one has:

Proposition $1 \Omega_{\text {Der }}(\mathfrak{A})=\Omega_{\text {Der,Bas }}(\mathfrak{B})$

Proof: The proof is based on results on noncommutative quotient manifolds studied in [7]. First, notice that $\mathfrak{A}$, as a set of section of an associated bundle of $P$, can be considered as the algebra $\left\{b \in \mathfrak{B} / A^{v} a+[A, a]=0 \forall A \in \mathfrak{s u}(n)\right\}$. Now, define as in $[7]$

$$
\begin{aligned}
\mathcal{Z}_{\text {Der }}(\mathfrak{A}) & =\{\mathfrak{X} \in \operatorname{Der}(\mathfrak{B}) / \mathfrak{X A}=0\} \\
\mathcal{N}_{\text {Der }}(\mathfrak{A}) & =\{\mathfrak{X} \in \operatorname{Der}(\mathfrak{B}) / \mathfrak{X A} \subset \mathfrak{A}\}
\end{aligned}
$$

Then looking locally (in a trivialization of $P$ ) at the derivations of $\mathfrak{B}$, one sees that

$$
\begin{aligned}
\mathcal{Z}(\mathfrak{A}) & =\mathfrak{A} \cap \mathcal{Z}(\mathfrak{B}) \\
\operatorname{Der}(\mathfrak{A}) & =\mathcal{N}_{\text {Der }}(\mathfrak{A}) / \mathcal{Z}_{\text {Der }}(\mathfrak{A}) \\
\mathfrak{A} & =\left\{b \in \mathfrak{B} / \mathfrak{X} b=0 \forall \mathfrak{X} \in \mathcal{Z}_{\text {Der }}(\mathfrak{A})\right\}
\end{aligned}
$$

where $\mathcal{Z}(\mathfrak{A})$ and $\mathcal{Z}(\mathfrak{B})$ are the center of the algebras $\mathfrak{A}$ and $\mathfrak{B}$ respectively. This makes $\mathfrak{A}$ into a noncommutative quotient manifold algebra of $\mathfrak{B}$ in the sense of [7]. In order to prove the proposition, using Prop V.1 in [7] and the fact that $\underline{\Omega}_{\text {Der }}$ and $\Omega_{\text {Der }}$ coincide in this context [5], it remains to show that the $\mathcal{Z}(\mathfrak{B})$-module induced by $\mathcal{N}_{\text {Der }}(\mathfrak{A})$ in $\operatorname{Der}(\mathfrak{B})$ is $\operatorname{Der}(\mathfrak{B})$ itself. As before, using local expressions of derivations, this can be checked easily.

As an example, let us consider a $S U(n)$-connection on $P$. Denote by $\omega$ its 1form on $P$. It was shown in [5] that there exists a corresponding noncommutative 1 -form $\alpha \in \Omega_{\operatorname{Der}}(\mathfrak{A})$. This form comes from a basic 1-form in $\Omega_{\text {Der,Bas }}(\mathfrak{B})$, which is nothing but $\omega-i \theta$, where $\theta \in \Omega_{\text {Der }}^{1}\left(M_{n}(\mathbb{C})\right)$ is the canonical 1-form defined in [3]. The basicity of this 1 -form is a consequence of properties of $\omega$ and $i \theta$, in particular the equivariance of $\omega$. 
Now, notice that the commutative algebra $\mathcal{F}(M)$ of smooth functions on $M$ and its de Rham complex $\Omega(M)$ are the basic subalgebra of $\mathcal{F}(P)$ and the basic subcomplex of $\Omega(P)$ for the operation of $\mathfrak{s u}(n)$ induced by $A \mapsto A^{v}$. This operation is itself the restriction of the operation of $\mathfrak{s u}(n)$ considered previously. Then, from this point of view, $\Omega_{\text {Der }}(\mathfrak{A})$ is a natural generalization of $\Omega(M)$ containing informations on $P$.

Moreover, this construction fits perfectly with the notion of noncommutative integration. It was shown in [3] that such a notion exists on the noncommutative differential calculus $\Omega_{\text {Der }}\left(M_{n}(\mathbb{C})\right)$. We denote by $\omega \in \Omega_{\text {Der }}^{n^{2}-1}\left(M_{n}(\mathbb{C})\right) \mapsto \int_{\text {nc }} \omega \in \mathbb{C}$ this noncommutative integral. This integral induces a map

$$
\begin{aligned}
\Omega_{\text {Der }}^{p, n^{2}-1}(\mathfrak{B}) & \rightarrow \Omega^{p}(P) \\
\omega & \mapsto \int_{\mathrm{nc}} \omega
\end{aligned}
$$

which satisfies:

Proposition 2 1) If $\omega \in \Omega_{\mathrm{Der}}^{p, n^{2}-1}(\mathfrak{B})$ is horizontal (resp. invariant), then $\int_{\mathrm{nc}} \omega \in$ $\Omega^{p}(P)$ is horizontal (resp. invariant) for the two operations defined above.

2) Considering basic elements, this map defines a canonical noncommutative integration "along the (noncommutative) fiber" $\Omega_{\text {Der }}(\mathfrak{A}) \rightarrow \Omega(M)$.

3) This noncommutative integral is compatible with the differentials:

$$
\int_{\mathrm{nc}} \hat{d} \omega=d \int_{\mathrm{nc}} \omega
$$

4) This induces maps in cohomologies:

$$
\begin{aligned}
& \int_{\mathrm{nc} *}: H^{r}\left(\Omega_{\text {Der }}(\mathfrak{B}), \hat{d}\right) \rightarrow H^{r-\left(n^{2}-1\right)}(P) \\
& \int_{\mathrm{nc} *}: H^{r}\left(\Omega_{\operatorname{Der}}(\mathfrak{A}), \hat{d}\right) \rightarrow H^{r-\left(n^{2}-1\right)}(M)
\end{aligned}
$$

Proof: 1) and 3) are straightforward computations using the precise definitions of the noncommutative integration over $\Omega_{\text {Der }}\left(M_{n}(\mathbb{C})\right)$, the differentials, and the two operations.

2) and 4) are immediate consequences of 1) and 3). 
The cohomology groups involved in 4) will be described in the next section where the computation of the cohomology of $\Omega_{\text {Der }}(\mathfrak{A})$ is performed.

The different relations between the various differential calculi can be summarized in the following diagram:

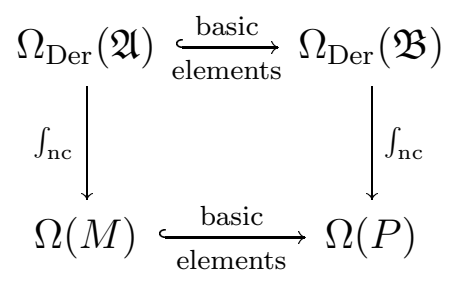

\section{The cohomology of $\Omega_{\text {Der }}(\mathfrak{A})$}

In the case when $\mathfrak{A}$ is a tensor product $\mathfrak{A}=\mathcal{F}(M) \otimes M_{n}(\mathbb{C})$, the cohomology of $\Omega_{\text {Der }}(\mathfrak{A})=\Omega(M) \otimes \Omega_{\text {Der }}\left(M_{n}(\mathbb{C})\right)$ is known [4]. It is just the tensor product of the cohomology of $\Omega(M)$ (the de Rham cohomology) and the cohomology of $\Omega_{\text {Der }}\left(M_{n}(\mathbb{C})\right.$ ) (we will detail this last cohomology in the following).

In the general case, the cohomology of $\Omega_{\text {Der }}(\mathfrak{A})$ can be computed using a slight modification of standard constructions in algebraic topology [1]. In this section, we perform this computation and show that the result is the same as in the tensor product case.

Let $U$ be a open subset of $M$ such that the restriction of $\operatorname{End}(E)$ to $U$ is trivial. We make a choice of trivializations for any such open subset and we denote by $\mathfrak{A}(U)$ the trivialization of the restriction to $U$ of the algebra $\mathfrak{A}$. Then one has $\mathfrak{A}(U) \simeq C^{\infty}(U) \otimes M_{n}(\mathbb{C})$. Denote by $g_{U V}: U \cap V \rightarrow S U(n)$ the transition functions.

Consider now the presheaf $\mathcal{F}$ defined by $U \mapsto \Omega_{\text {Der }}(\mathfrak{A}(U))$ where $U$ is any open subset of $M$ which trivializes $\operatorname{End}(E)$. For $V \subset U$, the inclusion map is defined to be

$$
\begin{aligned}
i_{U}^{V}: \Omega_{\operatorname{Der}}(\mathfrak{A}(U)) & \rightarrow \Omega_{\text {Der }}(\mathfrak{A}(V)) \\
\omega & \mapsto\left(\omega_{\mid V}\right)^{g_{U V}}
\end{aligned}
$$


where $\left(\omega_{\uparrow V}\right)^{g_{U V}}$ is the action of $g_{U V}$ (change of trivialization) on the restriction of $\omega$ to $V$. If $\omega=a_{0} \hat{d} a_{1} \ldots \hat{d} a_{p}$, one has

$$
\omega^{g_{U V}}=\left(g_{U V}^{-1} a_{0} g_{U V}\right) \hat{d}\left(g_{U V}^{-1} a_{1} g_{U V}\right) \ldots \hat{d}\left(g_{U V}^{-1} a_{p} g_{U V}\right)
$$

This action commute with $d$.

Now, let us take a good cover $\mathfrak{U}=\left\{U_{\alpha}\right\}_{\alpha \in I}$ of $M$ indexed by an ordered set $I$ and such that over any $U_{\alpha}, \operatorname{End}(E)$ is trivialized. For convenience, on any $U_{\alpha_{1} \ldots \alpha_{q}}=U_{\alpha_{1}} \cap \cdots \cap U_{\alpha_{q}}$ the trivialization is chosen to be the restriction of the trivialization of $U_{\alpha_{q}}$.

We now define a noncommutative version of the double Čech-de Rham complex associated with this presheaf. For $p \geq 0$ and $q \geq 0$, consider the vector spaces

$$
C^{p, q}(\mathfrak{U}, \mathcal{F})=\prod_{\alpha_{0}<\cdots<\alpha_{p}} \Omega_{\mathrm{Der}}^{q}\left(\mathfrak{A}\left(U_{\alpha_{0} \ldots \alpha_{p}}\right)\right)
$$

An element $\omega \in C^{p, q}(\mathfrak{U}, \mathcal{F})$ is a collection of noncommutative $q$-form in $\omega_{\alpha_{0} \ldots \alpha_{p}} \in$ $\Omega_{\text {Der }}^{q}\left(\mathfrak{A}\left(U_{\alpha_{0} \ldots \alpha_{p}}\right)\right)$.

Define the differential $\delta: C^{p, q}(\mathfrak{U}, \mathcal{F}) \rightarrow C^{p+1, q}(\mathfrak{U}, \mathcal{F})$ by

$$
(\delta \omega)_{\alpha_{0} \ldots \alpha_{p+1}}=\sum_{i=0}^{p}(-1)^{i} \omega_{\alpha_{0} \ldots \alpha_{i-1} \alpha_{i+1} \ldots \alpha_{p+1}}+(-1)^{p+1} \omega_{\alpha_{0} \ldots \alpha_{p}}^{g_{\alpha_{p} \alpha_{p+1}}}
$$

Using the properties of the transition functions it is easy to verify that $\delta^{2}=0$. The noncommutative differential $\hat{d}$ is of degree $(0,1)$ on this double complex and satisfies $\hat{d} \delta=\delta \hat{d}$. On the total complex of this double complex, we introduce the differential $D=\delta+(-1)^{p} \hat{d}$, with $D^{2}=0$.

For $p=-1$, define $C^{-1, q}(\mathfrak{U}, \mathcal{F})$ to be $\Omega_{\text {Der }}^{q}(\mathfrak{A})$, and $\delta: C^{-1, q}(\mathfrak{U}, \mathcal{F}) \rightarrow$ $C^{0, q}(\mathfrak{U}, \mathcal{F})$ to be the restriction map. Then $\delta^{2}=0$ also holds on $C^{-1, q}(\mathfrak{U}, \mathcal{F})$.

Lemma 3 The following sequence is exact :

$$
0 \longrightarrow C^{-1, *}(\mathfrak{U}, \mathcal{F}) \stackrel{\delta}{\longrightarrow} C^{0, *}(\mathfrak{U}, \mathcal{F}) \stackrel{\delta}{\longrightarrow} C^{1, *}(\mathfrak{U}, \mathcal{F}) \stackrel{\delta}{\longrightarrow} \cdots
$$

Proof: The exactitude at $C^{-1, *}(\mathfrak{U}, \mathcal{F})$ is trivial. Because $\delta^{2}=0$, one has only to show that if $\omega \in C^{p, *}(\mathfrak{U}, \mathcal{F})$, with $\delta \omega=0$, then there exists $\eta \in C^{p-1, *}(\mathfrak{U}, \mathcal{F})$ such 
that $\delta \eta=\omega$. Introduce $\left\{\rho_{\alpha}\right\}$ a partition of unity subordinate to the good cover U. For $\alpha_{0}<\cdots<\alpha_{p-1}$, define

$$
\eta_{\alpha_{0} \ldots \alpha_{p-1}}=\sum_{\substack{\alpha<\alpha_{p-1} \\ \alpha \neq \alpha_{0}, \ldots, \alpha_{p-2}}} \rho_{\alpha} \omega_{\alpha \alpha_{0} \ldots \alpha_{p-1}}+\sum_{\alpha>\alpha_{p-1}} \rho_{\alpha} \omega_{\alpha \alpha_{0} \ldots \alpha_{p-1}}^{g_{\alpha \alpha_{p}-1}}
$$

where, to simplify this expression, we make use of the notation $\omega_{\ldots \alpha_{i} \ldots \alpha_{j} \ldots}=$ $-\omega_{\ldots \alpha_{j} \ldots \alpha_{i} \ldots}$. Note that $\rho_{\alpha} \omega_{\alpha \alpha_{0} \ldots \alpha_{p-1}} \in \Omega_{\operatorname{Der}}\left(\mathfrak{A}\left(U_{\alpha \alpha_{0} \ldots \alpha_{p-1}}\right)\right)$. A straightforward computation shows then that with $\delta \omega=0$, one has $\delta \eta=\omega$.

Using general arguments on double complexes, this lemma gives us a noncommutative version of the Generalized Mayer-Vietoris Principle:

Corollary 4 The cohomology of $\left(\Omega_{\text {Der }}(\mathfrak{A}), \hat{d}\right)$ is the cohomology of the total complex $(C(\mathfrak{U}, \mathcal{F}), D)$.

Consider now the spectral sequence $\left\{E_{r}, d_{r}\right\}$ associated with the double complex $C(\mathfrak{U}, \mathcal{F})$, induced by the filtration

$$
F^{p} C(\mathfrak{U}, \mathcal{F})=\oplus_{s \geq p} \oplus_{q \geq 0} C^{s, q}(\mathfrak{U}, \mathcal{F})
$$

Then by standard argument, one knows that the first term of this spectral sequence is

$$
E_{1}^{p, q}=H_{\hat{d}}^{p, q}=C^{p}\left(\mathfrak{U}, \mathcal{H}^{q}\right)
$$

where $\mathcal{H}^{q}$ is the presheaf $\mathcal{H}^{q}(U)=H^{q}\left(\Omega_{\text {Der }}(\mathfrak{A}(U)), \hat{d}\right)$ and that the second term is

$$
E_{2}^{p, q}=H_{\delta}^{p}\left(\mathfrak{U}, \mathcal{H}^{q}\right)
$$

This spectral sequence converges to $H(C(\mathfrak{U}, \mathcal{F}), D)=H\left(\Omega_{\text {Der }}(\mathfrak{A}), \hat{d}\right)$.

The cohomology groups $H^{*}\left(\Omega_{\text {Der }}(\mathfrak{A}(U)), \hat{d}\right)$ have been computed $[2,3,4]$. When $U$ is diffeomorphic to $\mathbb{R}^{m}$, one has

$$
H^{*}\left(\Omega_{\operatorname{Der}}(\mathfrak{A}(U)), \hat{d}\right)=H^{*}\left(\Omega_{\operatorname{Der}}\left(M_{n}(\mathbb{C})\right), d^{\prime}\right)
$$


and $H^{*}\left(\Omega_{\operatorname{Der}}\left(M_{n}(\mathbb{C})\right), d^{\prime}\right)$ is isomorphic to the cohomology of the Lie algebra $\mathfrak{s l}(n, \mathbb{C})$. Then $H^{*}\left(\Omega_{\operatorname{Der}}\left(M_{n}(\mathbb{C})\right), d^{\prime}\right)=\left(\bigwedge \mathfrak{s l}(n, \mathbb{C})^{*}\right)_{\text {Inv }}$, where Inv denotes the invariant elements for the canonical Lie derivation. This algebra can also be considered as the graded commutative algebra freely generated by $c_{2 r-1}^{n}$ in degree $2 r-1$ with $r \in\{2,3, \ldots, n\}$ and where the $c_{2 r-1}^{n}$ are the primitive elements of $\left(\bigwedge \mathfrak{s l}(n, \mathbb{C})^{*}\right)_{\mathrm{Inv}}$.

Any element in $\left(\bigwedge \mathfrak{s l}(n, \mathbb{C})^{*}\right)_{\text {Inv }}$ is then invariant by the action of $S U(n)$. So, the inclusion map is the identity and then the presheaf $\mathcal{H}^{q}$ is a constant presheaf. The cohomology of $E_{1}$ reduces to the Čech cohomology of this constant presheaf. Now, using again the structure of $H^{*}\left(\Omega_{\operatorname{Der}}\left(M_{n}(\mathbb{C})\right), d^{\prime}\right)$, it is easy to see that the differentials $d_{2}, \ldots, d_{r}$ are all zero. We have then proven:

Proposition $5 E_{\infty}=E_{2}=H^{*}(M) \otimes\left(\bigwedge \mathfrak{s l}(n, \mathbb{C})^{*}\right)_{\mathrm{Inv}}$

This proposition tells us that the cohomology of $\Omega_{\text {Der }}(\mathfrak{A})$ is far more simpler that the cohomology of the underlying principal bundle.

Notice now that there is a canonical inclusion of algebras

$$
\left(\bigwedge \mathfrak{s l}(n, \mathbb{C})^{*}\right)_{\mathrm{Inv}} \hookrightarrow\left(\bigwedge \mathfrak{s l}(n+1, \mathbb{C})^{*}\right)_{\mathrm{Inv}}
$$

given by $c_{2 r-1}^{n} \mapsto c_{2 r-1}^{n+1}$ for $r \in\{2,3, \ldots, n\}$. Taking the inductive limit, one defines $\left(\bigwedge \mathfrak{s l}(\infty, \mathbb{C})^{*}\right)_{\text {Inv }}$, which is the graded commutative algebra freely generated by elements $c_{2 r-1}$ in degree $2 r-1$. Then, the cohomology of $\Omega_{\text {Der }}(\mathfrak{A})$ can be embedded in $H(M) \otimes\left(\bigwedge \mathfrak{s l}(\infty, \mathbb{C})^{*}\right)_{\text {Inv }}$, the right hand side being now completely independent of the oriented hermitian vector bundle $E$.

\section{Conclusions}

In this letter, we have added a few more arguments to those given in [5], to propose the algebra $\mathfrak{A}$ equipped with its noncommutative differential calculus based on derivations as a possible replacement of the principal bundle $P$. Indeed, we have shown that this noncommutative geometry of $\mathfrak{A}$ is strongly and very naturally related to the ordinary geometry of $P$. Then $\mathfrak{A}$ can be used in place of $P$, if one replaces the differential calculus $\Omega(P)$ by $\Omega_{\text {Der }}(\mathfrak{A})$, the connection 1 -form 
$\omega$ on $P$ by the associated noncommutative 1-form $\alpha$ introduced in [5] (which is only subjected to a "vertical" condition), the notion of associated vector bundle by the notion of (left-)module over $\mathfrak{A}$.

From a physical point of view, this noncommutative geometry is more interesting because, as was pointed out in $[4,5]$, it contains not only ordinary Yang-Mills fields, but also other fields which look very much like Higgs fields. On the other hand, from a mathematical point of view, this geometry looks simpler, in particular in cohomology.

\section{Acknowledgments}

We would like to thank Michel Dubois-Violette for very helpful discussions and his kind interest. 


\section{References}

[1] R. Bott, L. Tu: Differential Forms in Algebraic Topology, GMT 82, Springer-Verlag, 1982.

[2] M. Dubois-Violette: Dérivations et calcul différentiel non commutatif, C.R. Acad. Sci. Paris, t. 307, Série I, p. 403-408, 1988.

[3] M. Dubois-Violette, R, Kerner, J. Madore: Noncommutative Differential Geometry of Matrix Algebras, J. Math. Phys. 31, p. 316, 1990.

[4] M. Dubois-Violette, R, Kerner, J. Madore: Noncommutative differential geometry and new models of gauge theory, J. Math. Phys. 31, p. 323, 1990.

[5] M. Dubois-Violette, T. Masson: $S U(n)$-connections and noncommutative differential geometry, J. Geom. Phys. 25, n 1,2, p. 104, 1998.

[6] M. Dubois-Violette, P.W. Michor: Connections on central bimodules in noncommutative differential geometry, J. Geom. Phys. 20, p. 218, 1996.

[7] T. Masson: Submanifolds and Quotient Manifolds in Noncommutative Geometry, J. Math. Phys. 37, 5, p. 2484, 1996. 